\title{
Au miroir de l'hégélianisme. La phénoménologie « religieuse » de Michel Henry
}

Roberto Formisano

\section{(2) OpenEdition}

1 Journals

Édition électronique

URL : https://journals.openedition.org/alter/2016

DOI : 10.4000/alter.2016

ISSN : 2558-7927

Éditeur :

Association ALTER, Archives Husserl (CNRS-UMR 8547)

\section{Édition imprimée}

Date de publication : 1 novembre 2020

Pagination : 117-132

ISBN : 978-2-9550449-6-4

ISSN : $1249-8947$

\section{Référence électronique}

Roberto Formisano, "Au miroir de l'hégélianisme. La phénoménologie « religieuse » de Michel Henry », Alter [En ligne], 28 | 2020, mis en ligne le 22 décembre 2020, consulté le 14 octobre 2021. URL : http:// journals.openedition.org/alter/2016; DOI : https://doi.org/10.4000/alter.2016

Ce document a été généré automatiquement le 14 octobre 2021.

Revue Alter 


\title{
Au miroir de l'hégélianisme. La phénoménologie « religieuse » de Michel Henry
}

\author{
Roberto Formisano
}

\section{Introduction}

1 Le sujet de la présente étude s'inscrit dans le cadre d'une hypothèse de recherche visant les héritages de l'idéalisme allemand dans la philosophie phénoménologique française contemporaine. Il concerne plus particulièrement la première formation de la phénoménologie de Michel Henry, c'est-à-dire les années qui précèdent la publication de L'essence de la manifestation (1963) ${ }^{1}$, par rapport à l'hégélianisme du XX siècle.

Puisqu'il ne s'agit pas de faire un rapprochement simplement extérieur, voire artificiel, entre ces deux systèmes de pensées, la première partie de la présente étude fournira une reconstruction rapide du contexte herméneutique à l'intérieur duquel se situe ce lien, à vrai dire encore peu fréquenté par les interprètes, et dont les racines renvoient à un moment décisif de l'histoire de la philosophie française, à savoir les années 1930-1940. Il s'agit de l'époque où la première réception de la phénoménologie de Husserl et de Heidegger s'accompagna et se nourrit de la redécouverte des philosophies de l'idéalisme allemand ${ }^{2}$, et en particulier de Hegel et de ses écrits de jeunesse ${ }^{3}$. Sollicitée par son exigence fondamentale de s'émanciper des abstractions de sa tradition académique et métaphysique du XIX ${ }^{e}$ siècle, c'est par la «fusion» de ces horizons de débats (la Hegel-Renaissance ${ }^{4}$, la phénoménologie contemporaine, les philosophies de l'existence, la découverte des écrits de jeunesse de Marx, le néokantisme, etc.) que la philosophie française des années 1930-1940 découvre les moyens conceptuels pour réaliser son programme de « retour au concret » de l'expérience ${ }^{5}$.

À ce même ordre d'exigences théoriques et de sollicitations répond la philosophie henryenne naissante, dont la découverte de la phénoménologie de Husserl et de Heidegger a lieu dans le sillage de l'hégélianisme, de la phénoménologie et de 
l'existentialisme de son époque, en fonction de leur manière d'encadrer et de formuler les questions centrales concernant le phénomène de l'être et son rapport avec ce que Hegel appela, dans le sous-titre à sa Phénoménologie de l'esprit (1807), « l'expérience de la conscience».

Notre interprétation consiste à montrer que c'est en réagissant à l'hégélianisme que le jeune Michel Henry s'approprie, et ainsi hérite à sa manière, les catégories et l'encadrement général de la "problématique » fondatrice de sa phénoménologie dans L'essence de la manifestation. Sans pourtant vouloir minimiser l'importance de la médiation, cruciale et décisive, de la philosophie de Maine de Biran ${ }^{6}$ dans l'élaboration henryenne de sa philosophie de la subjectivité, la mise en valeur du renversement que Michel Henry opère à l'égard de l'hégélianisme nous servira de base pour poser en évidence certains "nœuds", ou plutôt certaines "tensions", internes à la conceptualisation henryenne de la phénoménologie et à son caractère "religieux ». L'analyse nous servira pour ouvrir, au moins, la question, décisive dans le cadre de notre recherche, concernant la détermination du sens propre au savoir phénoménologique et de son statut.

\section{Le contexte}

5 Au début de l'année académique 1946-1947, le jeune Michel Henry dépose auprès du CNRS de Paris sa première version d'un projet de recherche encore seulement esquissé ${ }^{7}$, mais qui, dix-sept ans plus tard, après plusieurs révisions et remaniements, changements de titres et modifications de structure, constituera dans sa version définitive le cœur de sa thèse d'État, publiée la première fois en deux tomes en 1963 et défendue en 1964, c'est-à-dire l'œuvre fondatrice de sa "phénoménologie de la vie »: L'essence de la manifestation.

6 La thèse principale, intitulée "Éléments pour une esthétique religieuse », est présentée sous la direction de Jean Wahl, interprète parmi les plus éminents de la philosophie de Kierkegaard en France ${ }^{8}$. Le choix de Jean Wahl comme directeur de recherche suggère une connexion significative entre le problème de l'« esthétique religieuse ", c'est-à-dire de l'expérience propre à la conscience religieuse, faite essentiellement de transcendance, de ruptures et de paradoxes, et la réception de la philosophie kierkegaardienne dans le cadre plus général de sa réaction contre l'esprit de système hégélien. Une série consistante de notes manuscrites de cette époque ${ }^{9}$ confirme en effet la centralité de la critique de l'hégélianisme dans l'œuvre de définition et de constitution du projet henryen, à la recherche de sa propre identité.

Chez Jean Wahl, cette connexion a constitué l'un des axes majeurs de son travail de réinterprétation de la Phénoménologie de Hegel. Avec son œuvre de 1929 consacré à la figure de la " conscience malheureuse » dans la philosophie de Hegel ${ }^{10}$, Wahl découvre ${ }^{11}$ chez le jeune Hegel, les mêmes tensions et les mêmes perplexités qui anticipent, de quelque manière, les critiques que Kierkegaard aurait ensuite adressées au philosophe de la Wissenschaft der Logik ou de l'Enzyclopädie, c'est-à-dire le Hegel de la maturité. En effet, le Hegel de Jean Wahl n'est pas le philosophe athée qui ouvre le chemin de Feuerbach et de Marx, mais le "traducteur " des thèmes principaux du christianisme dans le langage de la philosophie moderne. L'idée de la «mort de Dieu », chez le jeune Hegel, ne conduit pas à l'identification de l'Esprit avec l'homme et avec sa praxis, mais exprime plutôt, par l'événement de la Résurrection, la «christianisation » du monde, 
c'est-à-dire l'appropriation de celui-ci à la lumière de la tension que, dans la forme de la tradition judaïque-chrétienne, la conscience religieuse incarne à l'égard du transcendant. Le "malheur» de la conscience, c'est-à-dire l'expérience de l'«appropriation » de sa condition mortelle, devient pour Wahl la clé, non seulement pour réinterpréter la Phénoménologie et rendre " vivante ", en l'actualisant, la pensée de Hegel, mais aussi pour faire de cette philosophie "revitalisée» la base pour la compréhension d'autres programmes philosophiques de "retour au concret », dont la référence décisive aux années 1930 et suivantes est la phénoménologie de Husserl et de Heidegger.

$8 \quad$ L'ensemble de ces questions semblent être évoquées dans la liste bibliographique que Michel Henry joint à sa demande de bourse de 1947, et qui résume la constellation des références théoriques qui accompagne cette première phase de la recherche henryenne ${ }^{12}$. Dans ce cadre, au nom de Wahl, auquel s'associe celui, très important, de Jean Hyppolite (qui, après Martial Guéroult, deviendra directeur de thèse de Michel Henry, à partir de 1949-1950), s'ajoutent ceux « antinomiques », pour ainsi dire, parce que défenseurs d'une interprétation « athée » de l'hégélianisme, de Kojève et de Sartre.

9 L'interprétation de Kojève s'oppose à celle de Wahl en ceci que, d'après Kojève, la religion chez Hegel n'est qu'un "moment», bien que nécessaire, en vue d'un accomplissement de la philosophie hégélienne de l'esprit, dont la forme achevée consiste précisément dans la négation radicale de toute forme religieuse. De même que Wahl, Kojève n'hésite pas à considérer Hegel comme un « existentialiste » ante-litteram. Seul, cet encadrement, chez Kojève, ne vient plus d'une lecture préalable de Kierkegaard, mais de Heidegger. Le "malheur» de la conscience, le sentiment de l'angoisse qui traverse l'existence, n'est qu'un «moment», dont le sens originaire réside dans l'œuvre de la négativité.

10 Celle de Kojève est, certes, une interprétation créatrice de Hegel, et non philologique, dont l'influence a pourtant marqué en profondeur les développements postérieurs de la philosophie française ${ }^{13}$, s'il est vrai qu'il n'était "plus possible, en France du moins, d'accéder à Hegel sans faire le détour par Kojève $»^{14}$. De cette même médiation se ressent la première réception de la phénoménologie de Heidegger. L'Être et le Néant de Sartre, que Michel Henry déclare avoir étudié en 1947, en est un exemple significatif. Chez Sartre, on retrouve le thème hégélien de l'« en-soi » et du " pour-soi », réélaboré à partir de la conception kojèvienne du « Néant qui néantit » ${ }^{15}$.

11 Pour Kojève, cependant, la négativité, en ce qui concerne la réalité de l'homme - la "réalité humaine », c'est-à-dire le Dasein, d'après la première traduction française de cette notion fondamentale de l'ontologie phénoménologique heideggérienne - est et se réalise essentiellement dans et par le travail : l'homme n'est qu'action négatrice; une action qui, exercée d'abord sur la donnée immédiate de la conscience, transforme celleci, et ainsi la conserve en lui conférant une forme nouvelle. La négation est en ce sens une action créatrice. Son sens propre est celui de la dialectique, que Kojève renonce à appliquer à la nature et préserve uniquement pour la vie libre de l'esprit.

La découverte de la connexion entre la négativité et la liberté ${ }^{16}$ est le fait de la conscience malheureuse, qui pense l'homme à partir de sa création. Dans la tradition judéo-chrétienne, dont la figure de la conscience malheureuse est l'expression, l'homme avait été créé parfait ; après la "chute » dans le péché, il demeure pourtant dans la condition de pouvoir regagner sa perfection perdue, mais sous une autre forme. L'homme doit se faire autre. C'est pourquoi il est libre: précisément pour qu'il puisse se 
transformer, s'améliorer dans son essence, vivre pour un idéal à atteindre. Mais être libre, pour l'homme, signifie être mortel. Au contraire de la vie animale, qui finit simplement, la mort de l'homme est une suppression dialectique. L'homme sait qu'il doit mourir ; il est donc capable de transcender sa mort, de l'anticiper et de la dépasser par la médiation de la pensée : par son savoir, qui est aussi ce en quoi ce dépassement trouve son accomplissement. La vérité qui s'ensuit - la négation de la finitude constitutive de l'homme, la négation de sa mortalité - est le miroir dans et par lequel l'absolu prend une vision de soi : il se représente, se fait l'objet d'une représentation, et ainsi prend connaissance de soi, quoique sous une forme encore et toujours objectivée. En se reflétant, et en se faisant ainsi autre, dans le savoir humain, l'absolu se nie lui-même, et en même temps il demeure identique à soi. La Phénoménologie, en tant que description des modes d'existence humains ${ }^{17}$, est en effet un Discours sur l'Être, sur ses modes concrets de manifestation qui renvoient à la compréhension humaine du réel.

Dans son Introduction à la lecture de Hegel, Kojève explique que :

Pris isolément, le Sujet et l'objet sont des abstractions qui n'ont pas de « réalitéobjective » (Wirklichkeit), ni d'« existence-empirique » (Dasein). Ce qui existe en réalité, - du moment qu'il s'agit de la Réalité-dont-on-parle; et puisque nous parlons en fait de la réalité, il ne peut être question pour nous que d'une Réalitédont-on-parle ; ce qui existe en réalité, dis-je, c'est le Sujet-connaissant-l'objet ou, ce qui est la même chose, l'objet-connu-par-le-sujet ${ }^{18}$.

Pour Kojève, le Discours phénoménologique ne constitue rien d'étranger à la vérité de ce qui est « réel », rien de détaché de sa formation et de son devenir historique. Le Discours appartient à la réalité du Réel-vrai : il n'y a de réalité que dans la mesure où celleci se laisse décrire; la description est ce en quoi et par la médiation duquel le réel réalise effectivement, dans l'horizon du temps et de l'histoire, sa vérité. Le Discours est la négation de l'identité immédiate de l'être-substance; son action négatrice est le moteur de ce devenir phénoménal où s'avère reconstituée l'unité de la totalité de l'être (i.e., l'être en tant que totalité apprise dans le Discours, comme son objet discursif) ; la reconstitution de cette unité est ce en quoi l'être s'avère révélé à soi-même par la médiation $d u$ Discours. Ceci signifie, alors, que le Discours appartient au devenir phénoménal originaire de l'être, comme ce par la médiation duquel l'Être doit pouvoir accomplir sa manifestation, en se faisant autre-que-soi dans l'horizon temporellement fini du monde, par l'anthropogenèse s'opérant dans l'Histoire et par l'appropriation de ses "formes historiques ", à l'intérieur d'un processus dialectique dont la philosophie, se révélant comme l'auto-conscience de la conscience de soi de l'être, constitue le moment ultime.

\section{L’anti-hégélianisme de Michel Henry}

15 À la fin de sa première année de recherches, le jeune Henry modifie le titre de son projet principal, qui devient: "Négativité et Transcendance ${ }^{19}$. La question de la religion, initialement envisagée comme axe principal de sa thèse, est donc subsumée à l'intérieur de la question, plus large, qui concerne "l'expérience de la conscience ", c'est-à-dire son mouvement de «suppression dialectique » (Aufhebung). Dans une note préparatoire à L'essence de la manifestation consacrée à l'interprétation kojévienne de la dialectique de Hegel, Michel Henry écrit à cet égard :

"Vrai » = être-révélé-par-le-discours-de-sa-réalité.

Philosophie doit décrire non seulement l'Être mais encore l'être révélé, et rendre compte du fait de la révélation de l'Être par le Discours. 
Philosophie - totalité de ce qui est (être et discours, Substance et Sujet, Philosophie de la nature, Anthropologie). La philosophie du Sujet peut seulement parler de l'être-donné, mais de lui-même qui parle de l'être. Découverte de la catégorie de négativité et de la dialectique.

Le Discours est intégré à la totalité de l'Être, i.e. un monisme. ${ }^{20}$ l'hégélianisme "athée " de Kojève, que Michel Henry se sert de ses analyses. C'est plutôt en vertu d'une opposition radicale et consciente à l'égard de l'idée d'une unité constitutive entre la phénoménalité de l'Être et le Discours (à laquelle se trouve ainsi associé le souci pour le développement « athée » de la philosophie) que Michel Henry lit ses textes : l'apprentissage de leur théorie et de leurs catégories discursives se motive en raison de la volonté de s'approprier leurs fondements, afin de les détruire. Son parcours de recherche consiste donc dans la conceptualisation du principe fondamental sur lequel l'hégélianisme, l'existentialisme, et plus en général la phénoménologie contemporaine trouve son appui, sa condition ultime de possibilité.

Chez Kojève, Henry retrouve ce principe dans l'intégration que l'interprétation existentialiste de la dialectique suppose entre la phénoménalité de l'être et sa révélation par le discours phénoménologique. Cette intégration, qui consiste dans l'insertion du lien transcendantal que la phénoménologie reconnaît entre l'apparaître de l'être et la structuration de l'existence temporellement finie de la «réalité humaine ", caractérise la présupposition fondamentale à partir de laquelle devient possible la « conversion » de la Nichtigkeit de la conscience et de son expérience, à titre de détermination essentielle de l'être humain, voire de son existence, en mode constitutif de phénoménalisation de l'être en tant que tel. L'intégration des modes de compréhensions ontologiques propres à l'existence au sein de la vérité de l'être est ce que Michel Henry appelle dans ses notes "le monisme »: la réduction de ces deux processus différents à un seul mode de phénoménalisation, compris à la lumière de l'œuvre de la négativité, en tant que mode de structuration de la «transcendance horizontale » constitutive de l'expérience de la conscience par laquelle et dans laquelle a lieu l'événement de l'être, sa donation. Subsumée à l'intérieur du processus originaire de phénoménalisation de l'être, la transcendance de la conscience manifeste ainsi son caractère ontologique: elle le manifeste dans l'appropriation discursive de sa propre structure. Le discours phénoménologique, par lequel la conscience doit pouvoir achever la libération de sa propre essence, devient ainsi le lieu d'une libération ultérieure celle de l'être se révélant à soi-même, dans et par la Philosophie.

Un caractère essentiel de l'hégélianisme contemporain et de l'existentialisme consiste, certes, à ne pas céder à l'illusion propre au savoir absolu hégélien ${ }^{21}$. La réalisation à venir de la « suppression dialectique » (Aufhebung) ultime où la révélation à soi de l'être s'achève en fonction de la révélation à soi de la négativité (auto-conscience humaine), dont l'accomplissement est assuré par le Discours, dans le savoir philosophique, là où la Parole de l'Homme devient Parole de l'Être et où les deux s'identifient par la négation de leur différence préalable. Cependant, l'idée d'un lien structurel entre l'Être et la Réalité humaine est ce que les philosophies héritières de Hegel gardent de sa phénoménologie : l'Être, pour s'apparaître, doit pouvoir se différencier à l'égard de soi, s'extérioriser; il doit sortir de chez soi (an sich), et se faire autre que soi (für sich), s'objectiver dans l'horizon transcendantal de toute "vision" - i.e., compréhension possible de soi. Cet horizon est celui de l'existence, le Dasein ouvert, lui aussi, à la compréhension de son propre être, selon les modes propres à l'In-der-Welt-sein; d'où le 
maintien de l'idée d'une Zusammengehörigkeit, d'une coappartenance essentielle du Dasein au sein de la vérité de l'être.

La première section de L'essence de la manifestation donne une reconstruction détaillée et systématique de cette conception "moniste $»^{22}$ de l'être, dont la thèse caractéristique consiste à rendre la phénoménalité de l'être homogène à la structure ontologique de l'existence. Cette " homogénéité de l'être ${ }^{23}$ est ce qui fonde, dans la perspective de ce "monisme ", l'insertion des modes de compréhension "ontologique ", dont l'existence s'avère capable, à l'intérieur du mouvement phénoménal de l'être, de son apparaître: le savoir ontologique, bien qu'irréductiblement différent à l'égard du savoir absolu, est pourtant constitutivement intégré dans le processus originaire dans et par lequel l'être réalise son s'apparaître, c'est-à-dire son se-savoir-soi-même, dont le Discours phénoménologique est en quelque sorte l'achèvement.

\section{Aux sources de l'attitude « religieuse » de la phénoménologie de la vie}

Chez Henry, c'est l'essence, et non pas l'homme, qui est le sujet d'une "libération ». Contre l'idée moniste d'une intégration de l'être et de l'existence, la phénoménologie de Michel Henry revendique la Selbständigkeit de l'essence originaire, c'est-à-dire "l'autonomie » de l'absolu, son hétérogénéité à l'égard de la manifestation finie du Dasein et son indépendance à l'égard de ses modes d'appropriation et de compréhension propres à la conscience en général, ce qui signifie l'indépendance du savoir absolu à l'égard du Discours phénoménologique ${ }^{24}$, de son Concept $^{25}$.

21 La Selbständigkeit est une notion reprise de L'Être et le Néant de Sartre, et que Michel Henry utilise d'une manière significative et provocatrice. Chez Sartre, la Selbständigkeit désigne le caractère d'« autonomie » que la conscience naturelle attribue à la réalité, en tant qu' "être en soi», substance, réalité capable d'exister indépendamment de la conscience qui le contemple. Mais une conception semblable de la Selbständigkeit se retrouve aussi dans la Phénoménologie de Hegel, dans la figure de la conscience malheureuse, c'est-à-dire dans la compréhension religieuse de l'essence, qui non seulement considère cette dernière comme " autonome ", mais qui lui attribue aussi le caractère de l'ipséité, et ainsi reconnaît et projette, dans l'image de son Dieu transcendant, toutes les propriétés de perfection, éternité, infinité, etc. que la conscience se refuse à soi-même en raison de son être mortel.

La convergence entre la phénoménologie henryenne et la " conscience malheureuse " de Hegel n'est pourtant qu'une apparence, car la première s'oppose explicitement aux présuppositions régissant le «malheur » de la conscience religieuse hégélienne. Dans le contexte problématique de L'essence de la manifestation, la découverte de l'autonomie de l'essence est explicitement présentée à titre de détermination de l'essence qui n'est pas et ne peut pas être le résultat d'une Sinngebung, l'effet d'un acte thétique de la conscience. La Selbständigkeit est plutôt revendiquée à partir de la démonstration phénoménologique de l'impossibilité constitutive de l'essence interprétée au sens de la transcendance (comme horizon ultime de toute donation phénoménale) de s'assurer par elle-même la condition ultime de son propre apparaître ${ }^{26}$. À cette limite constitutive de la transcendance - limite « insurmontable » par l'analyse ${ }^{27}$ - s'associe la nécessité d'une radicalisation de la méthode phénoménologique qui, présentée comme 
"épochè du monde ", consiste dans la mise hors circuit de l'intentionnalité en tant que telle ${ }^{28}$. L'autonomie s'avère ainsi une détermination immanente à l'essence, s'imposant de soi, en vertu de sa capacité d'assurer par elle-même sa propre réceptivité, indépendamment de toute opérativité - active ou passive soit-elle - de la conscience à son égard. La compréhension henryenne de l'inessentialité de la conscience ontologique, ne fait pas problème ; elle ne s'accompagne pas du sentiment « douloureux » de la séparation avec l'essence, parce que cette séparation n'a jamais lieu : bien qu'inessentielle, en ce qui concerne ce que la conscience peut apporter à l'égard de mouvement originaire d'autorévélation de l'essence, la conscience s'avère fondée en celle-ci, d'une manière telle qu'aucune compréhension ou mécompréhension de la conscience ne peut ni modifier ni "rompre " ce lien que l'essence opère par elle-même, en elle-même, indépendamment de la conscience et de ses modes de compréhension de soi et de l'essence: "L'essence, pourtant ", Henry tient à le préciser, « est immanente à l'existence comme constituant son essence même ${ }^{29}$.

Le mot-clé, à cet égard, est celui d'immanence. "L'immanence - répète sans cesse Henry, dans L'essence de la manifestation - est l'essence de la transcendance »; elle est «le mode originaire selon lequel s'accomplit la révélation de la transcendance ellemême et, comme telle, l'essence originaire de la révélation $»^{30}$. L'immanence est selbständige parce que, afin d'accomplir son propre apparaître, elle n'a pas besoin de l'œuvre de la compréhension, n'exige pas la médiation de la conscience et de sa structure ontologique. En tant qu'immanence, l'essence n'est liée, quant à sa possibilité ultime de donation, à aucune " expérience de la conscience».

Ce que Michel Henry envisage, en affirmant l'« autonomie » de l'expérience purement passive et affective du "s'éprouver soi-même " propre à l'essence de la manifestation, est une disjonction essentielle ${ }^{31}$ entre le mode d'apparaître originaire, constitutif de l'autorévélation affective de l'essence réduite à la vie pure et simple, et le mode d'apparaître dérivé (et selon l'hégélianisme prétendument co-originaire au s'apparaître de l'essence) constitutif de la conscience et de son être-dans-le-monde ${ }^{32}$. Une disjonction qui ne veut pourtant pas dire une "séparation radicale» entre le s'apparaître originaire et affectif de la phénoménalité, que Michel Henry appelle la vie, et la structure ontologique de l'existence. En s'opposant à l'hégélianisme et à l'existentialisme, la phénoménologie de Michel Henry déclare le caractère inessentiel de toute intervention que la conscience peut opérer par son Verstehen en ce qui concerne le processus originaire à l'intérieur du quel s'accomplit l'autorévélation de la vie ${ }^{33}$. Le fait que l'existence soit " inessentielle » à l'égard de ce processus originaire, veut dire cependant que l'accomplissement "en soi » de ce dernier est ce qui constitue la condition ultime de possibilité pour l'ouverture de l'horizon transcendantal de l'être constituant la structure de l'existence, l'ouverture de son être-dans-le-monde. Autrement dit: c'est parce que l'autoaffection de la vie est déjà toujours donnée à elle-même, dans l'immédiation pathétique de son épreuve immanente de soi, que celle-ci se donne et se laisse trouver comme ce donné irréductible, purement " matériel », se présentant à la manière d'une réalité qui est "déjà là ", comme déjà donné ou pré-donné, et ainsi disponible aux prises de la conscience $^{34}$. L'immanence - répétons-le, avec Michel Henry - est l'essence de la transcendance.

Il s'ensuit que dans la perspective de Michel Henry, l'affirmation de l'« autonomie » de l'essence ne vise pas à nier le lien de fondation qui unit, au sein de la vie, la pensée et l'essence que celle-ci cherche à comprendre. Aucune négation n'est ici nécessaire parce 
qu'entre l'«autonomie » de l'essence et l'absence d'«autonomie » de la conscience il n'y a aucun conflit possible. Rien qui nécessite d'être "supprimé » dialectiquement. Puisque l'immanence fonde la transcendance, aucun travail (vs. Kojève), aucune maturation spirituelle de l'existence (vs. Wahl) n'est requise. En s'auto-interprétant comme déjà fondée dans la vie, par l'œuvre que celle-ci accomplit dans l'origine de son autorévélation immédiate et immanente, la conscience phénoménologique henryenne n'a pas besoin, pour être elle-même, de nier son attitude "religieuse " à l'égard de l'essence ${ }^{35}$. C'est plutôt en vertu de cette distinction que, de ce lien de fondation (ce religare immanent à la vie et à ses pouvoirs phénoménologiques), la phénoménologie henryenne vise à lui donner une signification nouvelle, alternative à celle réaffirmée par les phénoménologies issues de Hegel et reprise par les courants majeurs de la philosophie française contemporaine.

\section{Le problème « interne » de la philosophie}

À cette requalification des rapports entre la vie et l'existence de l'homme s'accompagne une révision des relations entre la vie et la pensée philosophique de la vie, c'est-à-dire entre l'essence et sa "phénoménologie première », car la vie, étant index sui, n'a pas besoin, en son mouvement originaire d'apparaître, d'être révélée par la médiation de la conscience en général, et en particulier par ce mode déterminé de son être dans le monde qui est la compréhension philosophique. «Il ne faut pas croire - écrit Henry dans une note préparatoire à L'essence de la manifestation - que notre vie est empirique et qu'il faut une opération exceptionnelle qu'il est réservé au philosophe d'accomplir, pour qu'elle [i.e., la vie] devienne une vie pure $» .{ }^{36}$ Dans la mesure où, cependant, la philosophie découvre le caractère inessentiel de sa médiation, en ce sens que ses descriptions et son discours ne constituent nullement une contribution "active " à l'accomplissement du processus originaire dans lequel elles donnent pourtant l'accès à la conscience phénoménologique, la question s'impose pour la philosophie de savoir à quel sens correspondent son exigence intime et ses efforts d'adhésion à la Sache selbst, à cette essence considérée en son mouvement constitutif d'autorévélation immanente.

Une fois acquise l'impossibilité de considérer le Discours phénoménologique comme un « moment » constitutif de l'apparaître originaire, Henry déclare que «si la philosophie est seconde par rapport à la vie, il doit cependant exister un mode de philosopher qui ne porte point de préjudice à l'essence $\|^{37}$. C'est pourquoi, en réfléchissant sur la tâche de la philosophie, par rapport à la vie, Henry écrit : « En vérité, le but de ce travail est de montrer qu'il existe une connaissance absolue et que celle-ci n'est pas solidaire d'un progrès quelconque. Une telle connaissance n'est pas liée, en effet, à un mode déterminé de l'existence, elle n'est pas le privilège d'un moment $\aleph^{38}$. Cette connaissance n'est pourtant pas celle de la philosophie : «Elle est plutôt le milieu même de l'existence, l'essence de la vie $»{ }^{39}$ Cette connaissance absolue, hétérogène à la phénoménalité de tout Verstehen en général, est celle de l'affectivité et de son autoaffection n'admettant aucune médiation extérieure, aucune intervention de la pensée en général. - Mais la philosophie, alors? Qu'en est-il du savoir phénoménologique s'obstinant à donner une description rigoureuse de cet apparaître « invisible » qui est le " s'éprouver soi-même » propre à la vie? Quel sens reconnaitre à ce travail qui la distingue de toute autre forme de savoir, et comment le légitimer? 
Henry n'ignore certes pas cette question ${ }^{40}$. Dans l'introduction à L'essence de la manifestation il déclare que « la signification ultime de la phénoménologie tient en ceci qu'elle est finalement la découverte d'un "phénomène" qui est le fondement luimême $»^{41}$. La question portant sur le sens à reconnaître à cette découverte et au travail d'éclaircissement que la phénoménologie opère en vue d'une saisie "rigoureuse " et «fidèle » à l'essence n'est pourtant même pas évoquée. Tout se passe comme si la simple volonté d'adhésion à l'essence, constitutive de la méthode phénoménologique, ne nécessite pas de justifications ultérieures; l'exclusion de la pensée hors du processus originaire où se réalise l'autoaffection de la vie absolue, est présentée comme l'indice d'une solution plutôt qu'une difficulté : finalement, c'est parce que la philosophie est seconde par rapport à la vie (parce qu'exclue de son mode originaire de donation), que l'accomplissement de cette dernière fonde la possibilité de sa description et fournit ainsi le critère pour savoir «quel mode de traitement phénoménologique il convient de faire subir au fondement $»^{42}$. En effet, c'est à partir de l'essence, et uniquement à partir d'elle, dans l'achèvement de cette phénoménalisation s'accomplissant en soi-même, que la phénoménologie revendique l'accès à la vie légitimant ses descriptions, c'est-àdire au moins la certitude pour le savoir phénoménologique de diriger ses visées vers une réalité "donnée en chair et os ", et de déterminer ainsi l'élément distinctif de la philosophie à l'égard de n'importe quel autre mode de compréhension.

30 Ici, cependant, une équivoque subtile semble s'insinuer car, ce n'est pas en raison de son adhérence à la donation de la vie que la philosophie s'avère légitimée en son ceuvre descriptive, mais seulement en raison de l'autonomie que in principio la phénoménalisation de la vie s'assure en elle-même. Légitime, en tant que savoir " ontologique », la philosophie l'est tout autant que n'importe quel autre savoir, comme par exemple le savoir naturel ${ }^{43}$. Savoir philosophique, savoir naturel : ces sont des modes d'être de l'existence dans le monde, auxquels la vie, en son mouvement pathétique immanent, s'avère indifférente ${ }^{44}$, parce que depuis toujours "présente à elle-même au sein d'une expérience transcendantale qui ne peut être à la rigueur ni "obtenue", ni "perdue" $»^{45}$. Le savoir philosophique est légitime dans la mesure où c'est à la vie d'en fonder la possibilité, sans que la philosophie - de même que n'importe quel autre mode de savoir - n'apporte quoi que ce soit à cette fondation.

31 Ce qui en général distingue la philosophie de tout autre mode d'existence est non seulement qu'elle exerce sa compréhension à l'égard de l'essence, mais aussi qu'elle sait, connaît et exprime par son langage la raison fondant la légitimité de sa parole : en elle s'accomplit l'auto-conscience de l'existence qui se veut «fidèle » à l'essence. Dans et par cette auto-conscience s'accomplit le travail critique que la philosophie, à titre de «critique de toute révélation ${ }^{46}$, dirige vers soi-même et vers son histoire. Mais comme cette auto-conscience s'avère hétérogène $e^{47}$, et donc extérieure, à l'égard de la vie, la question s'impose à nouveau de savoir en quoi doit pouvoir consister le sens de cette appropriation que la philosophie peut assurer à la fondation que la vie - et en aucune façon son auto-conscience - accomplit en son devenir phénoménal ? Puisqu'il n'est pas question, pour Henry de dépasser l'hétéronomie irréductible, voire religieuse, de la vie, il s'agit de savoir si la conscience de cette hétéronomie, bien qu'incapable d'apporter quelque chose de plus à la vie, pourrait apporter à la philosophie la détermination dont celle-ci a besoin - non pas pour la vie, mais pour elle-même, comme justificatif de son travail d'éclaircissement. Or, est-ce que le simple eidos de la vie - le seul eidos à laquelle la phénoménologie 
henryenne considère qu'il est légitime de faire appel - est capable d'assurer à la philosophie l'ensemble des conditions nécessaires pour répondre à cette question?

La question demeure, nous semble-t-il, tout à fait ouverte dans L'essence de la manifestation, où la stratégie argumentative de Michel Henry, centrée sur l'exigence de ne porter "point préjudices à l'essence ${ }^{48}$, consiste à laisser en arrière-plan le problème « interne » de la philosophie, celle-ci étant « seconde par rapport à la vie »ce qui veut dire : étrangère à l'ordre des questions propres à une "phénoménologie première ». Il se peut alors qu'une réponse puisse se trouver dans les œuvres postérieures à L'essence de la manifestation, où Michel Henry traite des œuvres d'art, de la critique de la culture, du problème de l'histoire et, notamment, de la possibilité d'une philosophie du christianisme. Il serait intéressant de vérifier, par exemple, si la " christologie phénoménologique " que Henry développe à partir de C'est moi la vérité (1996), dans le contexte des débats liés au «tournant théologique de la phénoménologie française $»^{49}$, apporte des solutions à ces difficultés théoriques: y-a-t-il eu effectivement un "tournant " radical en 1996, ou bien était-ce la conséquence des présupposés de la période de L'essence de la manifestation? Est-ce que le « tournant » des années 1990 constitue un retour au projet originaire d'élaboration d'une "esthétique religieuse ", ou en représente-t-il plutôt un développement ultérieur, sinon une sorte de dépassement?

Dans les limites de cette étude, visant d'abord la première formation philosophique de Michel Henry, on a montré que l'idée d'une " philosophie religieuse » est, en réalité, à l'origine de la démarche henryenne. Elle en est le but explicite en 1946; et même ensuite, lorsque la religion ne figure plus à titre de sujet principal et la recherche se consacre plutôt à l'élaboration d'une "phénoménologie première" à partir des possibilités ouvertes par la découverte du " corps subjectif », la philosophie henryenne ne modifie en rien son "attitude» qui domine sa compréhension de la méthode phénoménologique et accompagne sa critique dirigée contre le «monisme ontologique ». L'affirmation de la Selbständigkeit de la vie absolue et de son autoaffection se révèle cependant n'être pas immune de certaines apories que l'hégélianisme reconnaît comme caractéristiques de la pensée "religieuse » de l'essence. La mise en rapport de la phénoménologie henryenne avec l'hégélianisme - ne visant pas à suggérer un retour à ce dernier - nous a servi pour montrer la persistance d'une "tension", chez Henry, entre la vie et la pensée de la vie. Une tension que la phénoménologie henryenne refuse de reconnaître au sein de la vie elle-même ${ }^{50}$, et qu'elle confine au sein de la seule représentation philosophique de la vie. - Or c'est cette représentation qui est pourtant la phénoménologie henryenne elle-même.

\section{NOTES}

1. M. Henry, L'essence de la manifestation, Paris, PUF, 1963 (désormais : EM).

2. Cf. V. Descombes, Le Même et l'Autre, Paris, Minuit, 1979.

3. Cf. J. Wahl, Tableau de la philosophie française, Paris, Édition de la Revue Fontaine, 1946. 
4. Cf. G. Jarczyk, P.-J. Labarrière, « Cent-cinquante années de "réception" hégélienne en France ", Genèses 2, 1990, p. 109-130.

5. Cf. À cet égard les jugements de M. Merleau-Ponty, Sens et non-sens, Paris, Nagel, 1948, p. 158, 164, et de J. Hyppolite, Figures de la pensée philosophique, Paris, PUF, 1971, t. I, p. 233.

6. À Maine de Biran, Henry consacre son livre Philosophie et phénoménologie du corps, Paris, PUF, 1965. Dans l'avertissement à la seconde édition (1987), Henry déclare aussi : " Mon propos était alors d'établir contre l'idéalisme le caractère concret de la subjectivité ".

7. Il s'agit d'un dossier de recherche, rédigé manuellement, à partir de 1946, par Michel Henry, à l'époque jeune boursier au CNRS de Paris, en vue de son Doctorat-ès-lettres. La transcription de ce document a été publié dans J. Leclercq, C. Perrin (éd.), Genèse et structure de "L'essence de la manifestation", Paris, Hermann, 2017, p. 299-356 (désormais : Dossier CNRS).

8. Dossier CNRS, p. 303.

9. $C f$. Fonds d'archives Michel Henry (Plate-forme ALPhA, Université Catholique de Louvain), Ms. A 2033-6434.

10. J. Wahl, Le Malheur de la conscience dans la philosophie de Hegel, Paris, Alcan, 1929 ; voir aussi Études kierkegaardiennes, Paris, Aubier Montaigne, 1938.

11. En s'appuyant sur les textes de la période de Berne et de Francfort, cf. G.W.F. Hegel, Theologische Jugendschriften, Tübingen, Mohr, 1907.

12. Cf. Dossier CNRS, p. 309-310, où figurent, entre autres, les noms de Kierkegaard, Schelling, Jankélévitch, Hyppolite, Husserl, Heidegger, Sartre, accompagnés par les études de P. LachièzeRey et V. Jankélévitch consacrées à l'idéalisme allemand ; dans l'année suivante (octobre 1948), la liste des lectures effectuées s'enrichit significativement des noms de Böhme, Maître Eckhart, Fichte, Guéroult, Kojève, Maine de Biran, Merleau-Ponty, Niel, Nietzsche.

13. Cf. G. Canguilhem, "Hegel en France ", Revue d'histoire et de philosophie religieuse 4 (1948), p. 282-297.

14. J.-B. Vuillerod, « Hegel et ses ombres », Les Temps Modernes 4 (2017), p. 91.

15. A. Kojève, Introduction à la lecture de Hegel (1947), Paris, Gallimard, 1968, p. 574.

16. À la question de la liberté, Henry déclare en 1950 vouloir y consacrer « la sixième et dernière section » (Dossier CNRS, p. 330) de sa thèse. Dans EM, cette section ne figure pas. Une référence à la question de la liberté se trouve pourtant vers la fin de son introduction, où Henry spécifie, en note de bas de page, que « les analyses auxquelles il vient d'être fait allusion n'ont pu [...] trouver de place dans ce livre; elles feront l'objet de travaux ultérieurs» (EM, p. 58). Cependant, ces recherches sur la liberté n'ont jamais été publiées.

17. Cf. A. Kojève, op. cit., p. 39 sq.

18. Ibid., p. 451.

19. Dossier CNRS, p. 309.

20. Ms. A 2048.

21. Cf. J. Hyppolite, Genèse et structure de la "Phénoménologie de l'Esprit" de Hegel, Paris, AubierMontaigne, 1946, p. 197 sq. ; mais aussi son Introduction à la philosophie de l'histoire de Hegel (1948), Paris, Rivière, 1968, p. 51 sq.

22. Selon la définition que Michel Henry reprend de Kojève : $c f$. A. Kojève, op. cit., p. 485-486.

23. EM, p. 45.

24. On peut entrevoir ici, esquissés, les préalables généraux à la distinction entre la « Parole de Vie » et la «parole du monde » que Michel Henry explicitera dans sa «trilogie christologique » (1996-2002) ; cf. en particulier Parole du Christ, Paris, Seuil, 2002, chap. VII.

25. Cf. EM, \& 75, 77.

26. Cf. EM, § 27.

27. Cf. EM, p. 368 sq.

28. Cf. C. Serban, "Michel Henry et la question du fondement de l'intentionnalité », Bulletin d'Analyse Phénoménologique 6 (2010), p. 284-304. 
29. EM, p. 187.

30. EM, p. 280.

31. L'idée de cette "disjonction » est ce que Michel Henry retrouve dans la doctrine fichtéenne de la religion, cf. G. Rametta, "Michel Henry et le problème de la manifestation ", in R. Formisano et al. (éd.), Vivre la raison. M. Henry entre histoire des idées, philosophie transcendantale et nouvelles perspectives phénoménologiques, Paris, Vrin, 2016, p. 173-188 ; R. Formisano «L'eredità impossibile », Azimuth 6 (2018), p. 147-164.

32. Cf. EM, p. 203.

33. Cf. EM, p. 179.

34. Sur ce point se joue la différence essentielle avec la "phénoménologie hylétique» de Husserl : cf. M. Henry, Phénoménologie matérielle, Paris, PUF, 1990.

35. Cf. EM, p. 898.

36. Ms. A 5878.

37. EM, p. 56

38. EM, p. 55.

39. Idem.

40. Cf. EM, § 17 .

41. EM, p. 54 .

42. EM, p. 55. Cf. aussi M. Henry, Incarnation, Paris, Seuil, 2000, § 15.

43. Cf. EM, p. 180.

44. Cf. EM, p. 540, et notamment p. 563 sq. Cette indifférence est d'ailleurs ce qui constitue «l'essence du christianisme » (EM, p. 564).

45. EM, p. 54.

46. EM, p. 55.

47. Et ce, d'autant plus qu'elle interprète sa méthode - et ainsi s'auto-interprète elle-même comme « l'auto-explicitation de la vie transcendantale de la subjectivité absolue sous la forme de son auto-objectivation » (M. Henry, Phénoménologie matérielle, op. cit., p. 129).

48. EM, p. 56.

49. D'après la formule célèbre de D. Janicaud, Le tournant théologique de la phénoménologie française, Combas, L'Éclat, 1991.

50. En raison de l'idée que, à l'envers de la philosophie, la religion n'est ni un mode déterminé de l'existence, ni un mode du Verstehen. Comprise au sens « originaire » du religare, et ainsi dépourvue de toute "positivité ", la religion, chez Henry, s'avère assimilée à la vie, à son mouvement immanent d'autorévélation, qui est le seul et même processus par lequel la vie institue le lien de fondation avec chaque individu, chaque ego charnel constituant la communauté des vivants. C'est la vie qui fonde et garde l'unité de cette fondation, où aucune médiation extérieure - c'està-dire, la philosophie - ne peut rien. Cf. EM, § 49. 